\title{
VALUES OF BROWNIAN INTERSECTION EXPONENTS III: TWO-SIDED EXPONENTS
}

\author{
Gregory F. LAWLER ${ }^{\mathrm{a}}$, Oded SCHRAMM ${ }^{\mathrm{b}}$, Wendelin WERNER ${ }^{\mathrm{c}}$ \\ ${ }^{a}$ Department of Mathematics, Box 90320, Duke University, Durham, NC 27708-0320, USA \\ ${ }^{\mathrm{b}}$ Microsoft Corporation, One Microsoft Way, Redmond, WA 98052, USA \\ ${ }^{\mathrm{c}}$ Département de Mathématiques, Bât. 425, Université Paris-Sud, 91405 Orsay cedex, France
}

Received 17 October 2000, revised 30 January 2001

ABSTRACT. - This paper determines values of intersection exponents between packs of planar Brownian motions in the half-plane and in the plane that were not derived in our first two papers. For instance, it is proven that the exponent $\xi(3,3)$ describing the asymptotic decay of the probability of non-intersection between two packs of three independent planar Brownian motions each is $(73-2 \sqrt{73}) / 12$. More generally, the values of $\xi\left(w_{1}, \ldots, w_{k}\right)$ and $\tilde{\xi}\left(w_{1}^{\prime}, \ldots, w_{k}^{\prime}\right)$ are determined for all $k \geqslant 2, w_{1}, w_{2} \geqslant 1, w_{3}, \ldots, w_{k} \in[0, \infty)$ and all $w_{1}^{\prime}, \ldots, w_{k}^{\prime} \in[0, \infty)$. The proof relies on the results derived in our first two papers and applies the same general methods. We first find the two-sided exponents for the stochastic Loewner evolution processes in a half-plane, from which the Brownian intersection exponents are determined via a universality argument. @ 2002 Éditions scientifiques et médicales Elsevier SAS

RÉSUMÉ. - Nous déterminons dans cet article la valeur de certains exposants d'intersection entre mouvements browniens plans, qui n'étaient pas obtenus dans nos deux premiers articles. Par exemple, nous montrons que l'exposant $\xi(3,3)$ décrivant le comportement asymptotique de la probabilité de non-intersection entre deux paquets de trois mouvements browniens vaut (73$2 \sqrt{73}) / 12$. Plus généralement, les valeurs de $\xi\left(w_{1}, \ldots, w_{k}\right)$ et $\tilde{\xi}\left(w_{1}^{\prime}, \ldots, w_{k}^{\prime}\right)$ sont déterminées pour tout $k \geqslant 2, w_{1}, w_{2} \geqslant 1, w_{3}, \ldots, w_{k} \geqslant 0$ et $w_{1}^{\prime}, \ldots, w_{k}^{\prime} \geqslant 0$. ( 2002 Éditions scientifiques et médicales Elsevier SAS

\section{Introduction}

This paper is a follow-up to the papers [2,3], in which the exact values of many of the intersection exponents between planar Brownian motions were determined. It is assumed that the reader is familiar with the terminology and the results of [2,3], to which we also refer for background (in particular, the link with critical exponents for other models, such as critical percolation or self-avoiding walks in the plane) and a more complete bibliography.

E-mail addresses: jose@math.duke.edu (G.F. Lawler), schramm@Microsoft.com (O. Schramm), wendelin.werner@math.u-psud.fr (W. Werner). 
Let us first very briefly recall the definition of these intersection exponents. Suppose that $k \geqslant 2, n_{1}, \ldots, n_{k} \geqslant 1$ are integers, and that $\left(B^{l, j}\right)_{1 \leqslant l \leqslant k, 1 \leqslant j \leqslant n l}$ is a collection of independent planar Brownian motions started from distinct points in a half-plane $H$. Define the $k$ packs of Brownian motions $\mathfrak{B}^{l}(t):=\bigcup_{j=1}^{n_{l}} B^{l, j}[0, t], l=1,2, \ldots, k$. Consider the following events:

$$
\begin{aligned}
& \mathcal{E}(t)=\mathcal{E}_{\left(n_{1}, \ldots, n_{k}\right)}(t):=\bigcap_{1 \leqslant l<l^{\prime} \leqslant k}\left\{\mathfrak{B}^{l}(t) \cap \mathfrak{B}^{l^{\prime}}(t)=\emptyset\right\}, \\
& \widetilde{\mathcal{E}}(t)=\widetilde{\mathcal{E}}_{\left(n_{1}, \ldots, n_{k}\right)}(t):=\mathcal{E}_{\left(n_{1}, \ldots, n_{l}\right)}(t) \cap \bigcap_{l=1}^{k}\left\{\mathfrak{B}^{l}(t) \subset H\right\} .
\end{aligned}
$$

It is easy to see, using a subadditivity argument, that when $t \rightarrow \infty$,

$$
\mathbf{P}[\mathcal{E}(t)] \approx t^{-\xi / 2}, \quad \mathbf{P}[\widetilde{\mathcal{E}}(t)] \approx t^{-\widetilde{\xi} / 2},
$$

for some $\xi=\xi\left(n_{1}, \ldots, n_{k}\right)$ and $\widetilde{\xi}=\xi\left(n_{1}, \ldots, n_{k}\right)$, which are called the intersection exponents between $k$ packs of $\left(n_{1}, \ldots, n_{k}\right)$ Brownian motions in the plane and in the half-plane, respectively. Here, $f \approx g$ means $\lim _{t \rightarrow \infty} \log f / \log g=1$.

There exists natural extensions of $\xi$ and $\widetilde{\xi}$ to non-integer values of $n_{1}, \ldots, n_{k}$. For instance, one can define the exponents $\xi(1, w)$ and $\widetilde{\xi}(1, w)$ for all $w>0$ by the relations

$$
\begin{aligned}
& \mathbf{E}\left[\mathbf{P}\left[\mathcal{E}_{(1,1)}(t) \mid \mathfrak{B}^{1}(t)\right]^{w}\right] \approx t^{-\xi(1, w) / 2}, \\
& \mathbf{E}\left[\mathbf{P}\left[\widetilde{\mathcal{E}}_{(1,1)}(t) \mid \mathfrak{B}^{1}(t)\right]^{w}\right] \approx t^{-\widetilde{\xi}(1, w) / 2} .
\end{aligned}
$$

It is easy to see that these exponents $\widetilde{\xi}(1, w)$ and $\xi(1, w)$ coincide with the previously defined exponents when $w$ is a positive integer.

A second generalization are the two-sided exponents $\widetilde{\xi}(w, 1, w)$. One way to define them is as follows: Suppose that $k=3, n_{1}=n_{2}=n_{3}=1$, that $H$ is the upper halfplane $\mathbb{H}=\{x+\mathrm{i} y: y>0\}$, and that for all $l \in\{1,2,3\}, B^{l, 1}(0)=\mathrm{e}^{\mathrm{i} / \pi / 4}$ and define $\widehat{\mathfrak{B}}^{l}(t)=\left(0, \mathrm{e}^{\mathrm{i} l \pi / 4}\right] \cup B^{l, 1}[0, t]$ and the event $\widehat{\mathcal{E}}(t)$ that $\widehat{\mathfrak{B}}^{l}(t)$ for $l=1,2,3$ are disjoint subsets of the half-plane $H$. Loosely speaking, adding the segments $\left(0, \mathrm{e}^{\mathrm{i} l \pi / 4}\right]$ ensures that the three Brownian motions maintain their cyclic order around zero. Then $\widetilde{\xi}(w, 1, w)$ is defined for all $w>0$ by

$$
\mathbf{E}\left[\mathbf{P}\left[\widehat{\mathcal{E}}(t) \mid \mathfrak{B}^{2}(t)\right]^{w}\right] \approx t^{-\widetilde{\xi}(w, 1, w) / 2} .
$$

These exponents $\widetilde{\xi}(w, 1, w)$ coincide with the above definition when $w$ is an integer [5].

It has been shown in [5] that there exists a unique extension of $\xi$ and $\widetilde{\xi}$ to noninteger values of $n_{1}, \ldots, n_{k}$ that is symmetric in its arguments and satisfies the "cascade relations" (for all $1<j<k-1$ )

$$
\begin{aligned}
\xi\left(n_{1}, \ldots, n_{k}\right) & =\xi\left(n_{1}, \ldots, n_{j}, \widetilde{\xi}\left(n_{j+1}, \ldots, n_{k}\right)\right), \\
\widetilde{\xi}\left(n_{1}, \ldots, n_{k}\right) & =\widetilde{\xi}\left(n_{1}, \ldots, n_{j}, \widetilde{\xi}\left(n_{j+1}, \ldots, n_{k}\right)\right) .
\end{aligned}
$$


The extension of $\widetilde{\xi}$ is valid for all positive $n_{1}, \ldots, n_{k}$ while the extension of $\xi$ also requires that at least two of the arguments are greater or equal to 1 .

A first consequence [5] of these cascade relations is that the value of all extended exponents $\xi$ and $\widetilde{\xi}$ (and in particular their values when $w_{1}, \ldots, w_{k}$ are positive integers) can be expressed in terms of the functions $w \mapsto \xi(1,1, w), w \mapsto \widetilde{\xi}(1, w)$ and $w \mapsto$ $\widetilde{\xi}(w, 1, w)$ defined for all $w>0$.

A second consequence [5] is that the (extended) full-plane exponents are expressible as a function of the half-plane exponents

$$
\xi\left(w_{1}, w_{2}, \ldots, w_{k}\right)=\eta\left(\widetilde{\xi}\left(w_{1}, w_{2}, \ldots, w_{k}\right)\right),
$$

provided that $w_{1}, w_{2} \geqslant 1$; however, the function $\eta$ was not determined in [5].

Set

$$
U(x)=\sqrt{x+(1 / 24)}-\sqrt{1 / 24} .
$$

In [2], we determined the function $w \mapsto \widetilde{\xi}(1 / 3, w)$, and using the cascade relations (1.1) concluded that

$$
\widetilde{\xi}\left(w_{1}, w_{2}, \ldots, w_{k}\right)=U^{-1}\left(U\left(w_{1}\right)+U\left(w_{2}\right)+\cdots+U\left(w_{k}\right)\right)
$$

holds for all $k \geqslant 2$, all $w_{1}, w_{2}, \ldots, w_{k-1} \in\{p(p+1) / 6: p \in \mathbb{N}\}$, and all $w_{k}>0$. Equation (1.3) expands to

$$
\widetilde{\xi}\left(w_{1}, \ldots, w_{k}\right)=\frac{\left(\sqrt{24 w_{1}+1}+\sqrt{24 w_{2}+1}+\cdots+\sqrt{24 w_{k}+1}-(k-1)\right)^{2}-1}{24} .
$$

In [3], we showed that $\xi(1,1)=5 / 4$, determined the function $w \mapsto \xi(1,1,1, w)$, and concluded from the cascade relations and (1.3) that

$$
\forall x \geqslant 7 \quad \eta(x)=\frac{(\sqrt{24 x+1}-1)^{2}-4}{48} .
$$

Combined with (1.3) and (1.2), this gives the value of $\xi\left(w_{1}, \ldots, w_{k}\right)$ for a large collection of $w_{1}, \ldots, w_{k}$, but not for all of them.

In the present paper, we will prove the following results.

THEOREM 1.1. - The identity (1.3) holds for all $k \geqslant 2$ and for all $w_{1}, \ldots, w_{k} \geqslant 0$.

THEOREM 1.2. - The identity (1.4) holds for all $x \geqslant \widetilde{\xi}(1,1)=10 / 3$, so that for all $k \geqslant 2, w_{1}, w_{2} \geqslant 1$ and $w_{3}, \ldots, w_{k} \geqslant 0$,

$$
\begin{aligned}
\xi\left(w_{1}, \ldots, w_{k}\right) & =\eta \circ U^{-1}\left(U\left(w_{1}\right)+\cdots+U\left(w_{k}\right)\right) \\
& =\frac{\left(\sqrt{24 w_{1}+1}+\cdots+\sqrt{24 w_{k}+1}-k\right)^{2}-4}{48} .
\end{aligned}
$$

These two theorems determine almost all the Brownian intersection exponents. Those which they do not give are $\xi(n, w)$ where $n \in \mathbb{N}_{+}$and $w \in(0,1)$. It seems that the universality argument, which is used to translate information about $S L E_{6}$ exponents 
to Brownian exponents, cannot be extended to this range. Therefore, the techniques of $[2,3]$ and the present paper do not suffice.

To complete the picture, in the forthcoming paper [4], we determine these remaining exponents by analytic continuation. There, it will be shown that for integers $n \geqslant 1$, the mapping $w \mapsto \xi(n, w)$ is real-analytic in $(0, \infty)$. Combining this with the above theorems gives the value of $\xi(n, w)$ for all positive $w$ (i.e., removing the $w \geqslant 1$ condition), and gives the value of the disconnection exponents $\xi(n, 0):=\lim _{w \searrow 0} \xi(n, w)$ for all $n \in \mathbb{N}_{+}$. That is, (1.2) also holds when $k=2, w_{1} \in \mathbb{N}_{+}$and $0 \leqslant w_{2}<1$ and (1.4) holds for all $x \geqslant 1$. This will conclude the determination of all the two-dimensional Brownian intersection exponents that have been defined.

The proof of Theorem 1.1 is very similar to the proofs we used to derive (1.3) and (1.4) in [2,3]. A crucial role is played by the stochastic Loewner evolution process with parameter $6\left(S L E_{6}\right)$ introduced in [7]. In the present paper, first the two-sided exponents associated to $S L E_{\kappa}$ in a half-plane are computed. Then, via a universality argument, the values of the Brownian half-plane exponents $\widetilde{\xi}\left(1, w_{1}, 1, w_{2}\right)$ are deduced. This leads directly to Theorem 1.1 via the cascade relations satisfied by $\widetilde{\xi}$. Theorem 1.2 also immediately follows by using the results derived in [3].

Following is a rough and somewhat imprecise comparison of the approach used in the present paper in relation to those of [2] and [3]. In [2], we have studied the expectation of the derivative to any power $w \geqslant 0$ of a suitably normalized conformal map onto the complement of a chordal $S L E_{\kappa}$ process crossing a rectangle from left to right. The expectation was determined precisely. Its rate of decay as a function of the width corresponds to the exponent $\widetilde{\xi}(1 / 3, w)$. The reason that $1 / 3$ appears as the first argument, rather than 1 , is that the $S L E_{\kappa}$ process was permitted to touch one horizontal edge.

In the present paper, we study the expectation of an expression of the form $f^{\prime}\left(x_{1}\right)^{w_{1}} f^{\prime}\left(x_{2}\right)^{w_{2}}$, where $f$ is a suitably normalized conformal map. The points $x_{1}$ and $x_{2}$ at which these derivatives are computed are on the two sides of the $S L E_{\kappa}$ process, hence the name of the paper. The explicit formula for the expectation is not calculated, however, the decay rate as a function of the size of the $S L E_{\kappa}$ is determined, which suffices. The decay rate corresponds to the exponent $\widetilde{\xi}\left(w_{1}, 1, w_{2}\right)$. The calculation of the decay rate is via an eigenvalue computation, as in [3].

In [3], the decay rate of expectation of a single derivative raised to an arbitrary power $w_{1}>0$ was calculated for radial $S L E_{\kappa}$.

\section{Notations and terminology}

The present paper builds on the results of our previous papers [2,3], and it will be assumed that the reader is familiar with the terminology and tools used in these papers. In particular, we refer to these two papers for definitions and properties of chordal and radial $S L E_{6}$, Brownian excursions in a domain, and their relation to Brownian intersection exponents.

Let $f$ and $g$ be functions, and let $l \in \mathbb{R}$ or $l=\infty$. Say that $f(x) \sim g(x)$ when $x \rightarrow l$, if $f(x) / g(x) \rightarrow 1$. Write $f(x) \approx g(x)$, if $\log f(x) / \log g(x) \rightarrow 1$, and write 
$f(x) \asymp g(x)$, if $f(x) / g(x)$ is bounded above and below by positive finite constants when $x$ is sufficiently close to $l$.

For convenience, just as in $[5,6,2,3]$, we will use $\pi$-extremal distance, which is defined as $\pi$ times the usual extremal distance or extremal length in a domain. The $\pi$-extremal distance in a domain $D$ between two sets $A, A^{\prime}$ will be denoted $\ell\left(A, A^{\prime} ; D\right)$. For more information on extremal length, as well as other basic tools from complex analysis that we shall use (Koebe 1/4 Theorem, Schwarz Lemma), see, for instance, [1].

\section{Derivative $S L E_{\kappa}$ exponents}

Let $x \in(0,1)$, let $\kappa>0$, let $K_{t}$ be the hulls of chordal $S L E_{\kappa}$ in $\mathbb{H}$, from $x$ to $\infty$, and let $g_{t}: \mathbb{H} \backslash K_{t} \rightarrow \mathbb{H}$ be the conformal maps normalized by the hydrodynamic normalization $\lim _{z \rightarrow \infty} g_{t}(z)-z=0$. In other words, for all $z \in \mathbb{H}, g_{t}(z)$ is the solution of the ordinary differential equation

$$
\partial_{t} g_{t}(z)=\frac{2}{g_{t}(z)-W_{t}}, \quad g_{0}(z)=z,
$$

where $t \mapsto W_{t / \kappa}$ is a standard real-valued Brownian motion started from $W_{0}=x$. The set $\mathbb{H} \backslash K_{t_{0}}$ consists of all $z \in \mathbb{H}$ such that $t \mapsto g_{t}(z)$ is well-defined at least up to time $t_{0}$. Then, $\left(K_{t}, t \geqslant 0\right)$ is an increasing family of subsets of $\mathbb{H}$ : For more details on the definition of $\left(K_{t}, t \geqslant 0\right)$, some of its properties such as scaling, conformal invariance, see $[7,2]$.

Let

$$
T:=\inf \left\{t>0:\{0,1\} \cap \overline{K_{t}} \neq \emptyset\right\}
$$

denote the first time at which the $S L E_{\kappa}$ swallows 0 or 1 , and $T:=\infty$ if no such time exists. For all $t<T$, let

$$
f_{t}(z):=\frac{g_{t}(z)-g_{t}(0)}{g_{t}(1)-g_{t}(0)}
$$

be the conformal map from $\mathbb{H} \backslash K_{t}$ onto the upper half-plane such that $f_{t}(0)=0$, $f_{t}(1)=1$ and $f_{t}(\infty)=\infty$. Note that $f_{t}^{\prime}(\infty)=\left(g_{t}(1)-g_{t}(0)\right)^{-1}$ is decreasing and continuous in $t$ for $t<T$. Let $S:=-\lim _{t \nearrow T} \log f_{t}^{\prime}(\infty)$. Perform a time-change as follows: For all $s \in[0, S)$, define

$$
t(s):=\inf \left\{t \in[0, T): f_{t}^{\prime}(\infty) \leqslant \mathrm{e}^{-s}\right\}
$$

and the inverse map

$$
s(t):=-\log f_{t}^{\prime}(\infty)
$$

for all $t \in[0, T)$. For all $t<T$ define also

$$
Y_{s(t)}=Z_{t}:=\frac{W_{t}-g_{t}(0)}{g_{t}(1)-g_{t}(0)} .
$$

(This $Z_{t}$ was already used in [2].) Loosely speaking, $Y_{s}$ and $Z_{t}$ correspond to the image (under $f_{t}$ ) of the point where $K_{t}$ grows at time $t$. 
For all $s<S$, also set

$$
\alpha(s):=-\log f_{t(s)}^{\prime}(0), \quad \beta(s)=-\log f_{t(s)}^{\prime}(1) .
$$

For every $w_{1}, w_{2}>0$ and every smooth function $F:[0,1] \rightarrow[0,1]$, let

$$
h_{F}(x, s)=h_{F}\left(x, s, w_{1}, w_{2}\right):=\mathbf{E}_{x}\left[1_{\{s<S\}} F\left(Y_{s}\right) \exp \left(-w_{1} \alpha(s)-w_{2} \beta(s)\right)\right],
$$

where $\mathbf{E}_{x}$ refers to expectation with respect to the $S L E_{\kappa}$ started at $x$; that is, $W_{0}=Z_{0}=$ $Y_{0}=x$. In particular, write $h_{1}$ in case $F$ is the constant function 1 . That is,

$$
h_{1}(x, s)=\mathbf{E}_{x}\left[1_{\{t(s)<T\}} f_{t(s)}^{\prime}(0)^{w_{1}} f_{t(s)}^{\prime}(1)^{w_{2}}\right]
$$

THEOREM 3.1. - For all $w_{1}, w_{2}>0$ and $\kappa>0$, there exists some $c>0$ such that for all $x \in(0,1)$ and all $s \geqslant 1$

$$
G(x) \exp (-\lambda s) \leqslant h_{1}(x, s) \leqslant c G(x) \exp (-\lambda s)
$$

where

$$
\begin{aligned}
\lambda & =\lambda_{\kappa}\left(w_{1}, w_{2}\right) \\
& :=\frac{\left(\sqrt{(\kappa-4)^{2}+16 \kappa w_{1}}+\sqrt{(\kappa-4)^{2}+16 \kappa w_{2}}+\kappa\right)^{2}-(8-\kappa)^{2}}{16 \kappa}, \\
G(x) & :=x^{a_{1}}(1-x)^{a_{2}}, \quad a_{j}:=\frac{\kappa-4+\sqrt{(4-\kappa)^{2}+16 w_{j} \kappa}}{2 \kappa}, \quad j=1,2 .
\end{aligned}
$$

Remark. - It can be shown that this theorem also holds when $w_{1}=0$ and/or $w_{2}=0$, but this will not be done here.

Proof. - This is a first eigenvalue computation, and the proof will follow quite closely the proof of Lemma 3.2 in [3] (which is the corresponding result for radial $S L E_{\kappa}$, but with the derivative computed at only one point). A simple computation (using the definitions of $\alpha, \beta, Y, g_{t}$ and $\left.s(t)\right)$ shows that for all $s<S$,

$$
\mathrm{d} Y_{s}=\sqrt{\frac{\kappa Y_{s}\left(1-Y_{s}\right)}{2}} \mathrm{~d} B_{s}+\left(1-2 Y_{s}\right) \mathrm{d} s,
$$

where $B$ is a standard Brownian motion. Note also that $S$ is the first time at which $Y$ hits $\{0,1\}$ (unless $S=T=\infty$ ), and that

$$
\partial_{s} \alpha(s)=1 / Y_{s}, \quad \partial_{s} \beta(s)=1 /\left(1-Y_{s}\right) .
$$

We first use this to prove that

$$
h_{G}(x, s)=\exp (-\lambda s) G(x) .
$$


Let $X=[0,1] \times[0, \infty)$. Set $h=h_{G}$ and let $\widehat{h}(x, s)=\exp (-\lambda s) G(x)$. Observe that

$$
Q_{s}:=h\left(Y_{s}, s_{0}-s\right) \exp \left(-w_{1} \alpha(s)-w_{2} \beta(s)\right)
$$

is a local martingale on $s \leqslant s_{0}$. (For this, the choice of $G$ is not important.) Moreover, $h$ is smooth in $(0,1) \times(0, \infty)$. Consequently, the $\mathrm{d} s$ term in Itô's formula for $\mathrm{d} Q_{s}$ must vanish; that is,

$$
\partial_{s} h=(1-2 x) \partial_{x} h+(1-x) x \frac{\kappa}{4} \partial_{x}^{2} h-\left(\frac{w_{1}}{x}+\frac{w_{2}}{1-x}\right) h .
$$

It is immediate to verify that $\widehat{h}$ satisfies this differential equation in the interior of $X$. It is also clear that $\widehat{h}=h$ on $\partial X$.

In a moment, we shall see that $h$ is continuous in $X$. Assuming this for now, an easy application of the maximum principle gives $h=\widehat{h}$. Indeed, let $\varepsilon>0$, and suppose that there is some point $\left(x_{0}, s_{0}\right)$ with $h-\widehat{h} \geqslant \varepsilon$. Among all such points, choose one with $s_{0}$ minimal. Since $h=\widehat{h}$ on $\partial X,\left(x_{0}, s_{0}\right)$ must be in the interior. By minimality of $s_{0}$, it follows that $\partial_{s} h\left(x_{0}, s_{0}\right)-\partial_{s} \widehat{h}\left(x_{0}, s_{0}\right) \geqslant 0$ and that $h\left(x, s_{0}\right)-\widehat{h}\left(x, s_{0}\right)$ has a local maximum at $x_{0}$. From the latter fact, we may deduce that $\partial_{x}(h-\widehat{h})=0$ and $\partial_{x}^{2}(h-\widehat{h}) \leqslant 0$ at $\left(x_{0}, s_{0}\right)$. However, these facts put together contradict (3.4), and we may conclude that $h \leqslant \widehat{h}+\varepsilon$. The same argument shows that $h \geqslant \widehat{h}-\varepsilon$. Since $\varepsilon>0$ was arbitrary, it follows that $\widehat{h}=h$.

To establish (3.3), it therefore remains to prove the continuity of $h$. Suppose that $Y$ starts at $Y_{0}=x$ where $0<x<2^{-n_{0}-2} \min \left\{s_{0}, 1\right\}$, for some constants $s_{0}>0$ and $n_{0} \in \mathbb{N}_{+}$. Define the stopping times $v_{0}=0$ and for all $n \geqslant 0$,

$$
v_{n+1}:=\inf \left\{s>v_{n}: s=v_{n}+Y_{v_{n}} \text { or }\left|Y_{s}-Y_{v_{n}}\right| \geqslant Y_{v_{n}} / 2\right\} .
$$

Note that for all $n \leqslant n_{0}-1,0<Y_{v_{n}} \leqslant 2^{n} x, v_{n+1} \leqslant \sum_{j=0}^{n} Y_{v_{j}} \leqslant 2^{n+1} x$, so that $v_{n_{0}} \leqslant s_{0}$. Let $\mathcal{R}_{n}$ denote the event

$$
\mathcal{R}_{n}:=\left\{v_{n}=v_{n-1}+Y_{v_{n}}\right\} .
$$

Let $\mathcal{F}_{n}$ denote the $\sigma$-field generated by the events $\mathcal{R}_{1}, \ldots, \mathcal{R}_{n}$. There is a $c>0$ such that for $Y_{s}<1 / 2$, the diffusion term in (3.1) is bounded below by $c \sqrt{Y_{s}}$, and the drift term is bounded by 1 . Hence, it is not difficult (for instance, using Girsanov's formula and Doob's inequality) to see that for all $n \leqslant n_{0}$, the conditional probability $\mathbf{P}\left[\mathcal{R}_{n} \mid \mathcal{F}_{n-1}\right]$ is bounded below by a positive constant, which does not depend on $x$ and $n$. On the event $\mathcal{R}_{n}$, we have $\alpha\left(v_{n}\right)-\alpha\left(v_{n-1}\right) \geqslant 2 / 3$, by (3.2). It follows easily that $\alpha\left(s_{0}\right)$ tends in probability to $\infty$ when $x \searrow 0$, and therefore (since $w_{1}>0$ ) that $h$ tends to zero as $x \searrow 0$ (uniformly for $s \geqslant s_{0}$ ). A similar argument shows that $h \rightarrow 0$ as $x \nearrow 1$. It is easy to verify that for any $\varepsilon>0, h(x, s) \rightarrow G(x)$ as $s \rightarrow 0$ uniformly with respect to $x \in(\varepsilon, 1-\varepsilon)$. It is also easy to check that $h(x, s) \rightarrow 0$ when $(x, s) \rightarrow(0,0)$ or $(x, s) \rightarrow(1,0)$ (note that $G(0)=G(1)=0$ ). This shows that $h$ is continuous in $X$ and concludes the proof of (3.3).

Since $G \leqslant 1$, it is clear that for all $s>0$ and $x \in(0,1)$,

$$
h_{1}(x, s) \geqslant h_{G}(x, s)=\mathrm{e}^{-\lambda s} G(x) .
$$


It remains to prove that $\inf _{x \in(0,1)} \inf _{s \geqslant 1} h_{G}(x, s) / h_{1}(x, s)>0$. The Markov property at time $s-1$ shows that it suffices to establish this for $s=1$. Since both $h_{1}(\cdot, 1)$ and $h_{G}(\cdot, 1)$ are positive and continuous on $(0,1)$, it suffices to prove this when $x$ is close to 0 and close to 1 . By symmetry, it is enough to treat the case where $x$ is close to 0 . Now assume that $0<x \leqslant 1 / 2$. For every positive integer $n$, let

$$
r_{n}:=\inf \left\{h_{G}(x, s) / h_{1}(x, s): x \in\left[4^{-n}, 1 / 2\right], s \in\left[1-2^{-n}, 1\right]\right\} .
$$

Assume $4^{-n} \leqslant x<4^{-n+1}, 1-2^{-n} \leqslant s \leqslant 1$, and let

$$
\tau:=\inf \left\{s: Y_{s} \in\left\{0,4^{-n+1}\right\}\right\} .
$$

Note that (3.2) gives

$$
\mathbf{E}_{x}\left[\mathrm{e}^{-w_{1} \alpha\left(2^{-n}\right)} 1_{\left\{\tau>2^{-n}\right\}}\right] \leqslant \mathrm{e}^{-w_{1} 2^{n-2}} .
$$

Since $h_{1}(x, s) \geqslant h_{G}(x, s) \geqslant c x^{a_{1}}$ for some constant $c>0$ and all $(x, s)$ as chosen above, it follows that

$$
\mathbf{E}_{x}\left[\mathrm{e}^{-w_{1} \alpha(s)-w_{2} \beta(s)} 1_{\left\{s<S, \tau \leqslant 2^{-n}\right\}}\right] \geqslant\left(1-\varepsilon_{n}\right) \mathbf{E}_{x}\left[\mathrm{e}^{-w_{1} \alpha(s)-w_{2} \beta(s)} 1_{\{s<S\}}\right],
$$

where $\varepsilon_{n}:=c^{-1} 4^{n a_{1}} \mathrm{e}^{-w_{1} 2^{n-2}}$. However, since $s-\tau \geqslant 1-2^{-n+1}$ on the event $\left\{\tau \leqslant 2^{-n}\right\}$, and since $\left\{s<S, \tau \leqslant 2^{-n}\right\} \subset\left\{Y_{\tau}=4^{-n+1}\right\}$, the strong Markov property gives

$$
\begin{aligned}
h_{G}(x, s) & \geqslant \mathbf{E}_{x}\left[\mathrm{e}^{-w_{1} \alpha(s)-w_{2} \beta(s)} G\left(Y_{s}\right) 1_{\left\{s<S, \tau \leqslant 2^{-n}\right\}}\right] \\
& =\mathbf{E}_{x}\left[\mathrm{e}^{-w_{1} \alpha(\tau)-w_{2} \beta(\tau)} h_{G}\left(4^{-n+1}, s-\tau\right) 1_{\left\{\tau \leqslant 2^{-n}, Y_{\tau}=4^{-n+1}\right\}}\right] \\
& \geqslant r_{n-1} \mathbf{E}_{x}\left[\mathrm{e}^{-w_{1} \alpha(\tau)-w_{2} \beta(\tau)} h_{1}\left(4^{-n+1}, s-\tau\right) 1_{\left\{\tau \leqslant 2^{-n}, Y_{\tau}=4^{-n+1}\right\}}\right] \\
& =r_{n-1} \mathbf{E}_{x}\left[\mathrm{e}^{-w_{1} \alpha(s)-w_{2} \beta(s)} 1_{\left\{s<S, \tau \leqslant 2^{-n}\right\}}\right] \\
& \geqslant r_{n-1}\left(1-\varepsilon_{n}\right) \mathbf{E}_{x}\left[\mathrm{e}^{-w_{1} \alpha(s)-w_{2} \beta(s)} 1_{\{s<S\}}\right] \\
& =r_{n-1}\left(1-\varepsilon_{n}\right) h_{1}(x, s) .
\end{aligned}
$$

That is, $r_{n} \geqslant\left(1-\varepsilon_{n}\right) r_{n-1}$. Since $\sum_{n} \varepsilon_{n}<\infty$, this gives $\inf _{n} r_{n}>0$, which completes the proof.

\section{Extremal distance exponents}

In the previous section, we derived estimates concerning the joint $\operatorname{law}$ of $\log f^{\prime}(0)$ and $\log f^{\prime}(1)$ at the first time at which $f_{t}^{\prime}(\infty)=\mathrm{e}^{-s}$. We now use this result to obtain information concerning the law of the extremal distances at the first time at which $S L E_{\kappa}$ reaches distance $R$. More precisely, let $R \geqslant 1$, and let $V_{R}$ denote the half disk

$$
V_{R}:=\{z \in \mathbb{H}:|z-1 / 2|<R\} .
$$

Let $A_{R}$ denote the semi-circle $\mathbb{H} \cap \partial V_{R}$. Let $a \in(0,1)$, and consider chordal $S L E_{\kappa}$ in $\mathbb{H}$ from $a$ to $\infty$. Let

$$
\tau=\tau_{R}:=\inf \left\{t: \bar{K}_{t} \cap A_{R} \neq \emptyset\right\},
$$


and set

$$
\mathfrak{K}=\mathfrak{K}_{R}:=\bigcup_{t<\tau} K_{t} .
$$

As before, let $T$ be the first time that the $S L E_{\kappa}$ swallows 0 or 1 . Let $I_{1}(t):=[0, a] \backslash \bar{K}_{t}$ and $I_{2}(t):=[a, 1] \backslash \bar{K}_{t}$. On the event $\tau<T$, let $\mathfrak{L}_{1}(R):=\ell\left(I_{1}(\tau), A_{R} ; \mathbb{H} \backslash \mathfrak{K}\right)$ denote the $\pi$-extremal distance from $I_{1}(\tau)$ to $A_{R}$ in $\mathbb{H} \backslash \mathfrak{K}$ (or in $V_{R} \backslash \mathfrak{K}$ since they are equal), and let $\mathfrak{L}_{2}(R):=\ell\left(I_{2}(\tau), A_{R} ; \mathbb{H} \backslash \mathfrak{K}\right)$. Let

$$
H(a, R):=\mathbf{E}_{a}\left[1_{\{\tau<T\}} \exp \left(-w_{1} \mathfrak{L}_{1}(R)-w_{2} \mathfrak{L}_{2}(R)\right)\right]
$$

THEOREM 4.1. - Let $\kappa>0, w_{1}, w_{2}>0$, and let $\lambda=\lambda_{\kappa}\left(w_{1}, w_{2}\right)$ be as in Theorem 3.1. There is a constant $c=c\left(\kappa, w_{1}, w_{2}\right)$ such that for all $R>2$,

$$
\forall a \in(0,1) \quad H(a, R) \leqslant c R^{-\lambda} .
$$

On the other hand, for all $a_{0} \in(0,1 / 2)$, there is a $c^{\prime}=c^{\prime}\left(\kappa, w_{1}, w_{2}, a_{0}\right)>0$ such that for all $R>2$

$$
\forall a \in\left[a_{0}, 1-a_{0}\right] \quad H(a, R) \geqslant c^{\prime} R^{-\lambda} .
$$

Proof. - We use the notation of Section 3. Using scaling invariance and a monotonicity argument, it is easy to see that for all $R>2$ and $a \in\left[a_{0}, 1-a_{0}\right]$,

$$
H\left(1 / 2,(R+1) / a_{0}\right) \leqslant H(a, R) \leqslant H(1 / 2,(R-1) / 2),
$$

and hence it suffices to show that $H(1 / 2, R) \asymp R^{-\lambda}$.

The Koebe $1 / 4$ Theorem implies that if $F: D_{1} \rightarrow D_{2}$ is a conformal transformation with $F(0)=0$ and $r_{j}:=\operatorname{dist}\left(0, \partial D_{j}\right)<\infty$, then

$$
\frac{r_{2}}{4 r_{1}} \leqslant\left|F^{\prime}(0)\right| \leqslant \frac{4 r_{2}}{r_{1}}
$$

Applying the Koebe $1 / 4$ Theorem again, using the estimate on $\left|F^{\prime}(0)\right|$, gives

$$
\frac{r_{2}}{16 r_{1}}|z| \leqslant|F(z)| \leqslant \frac{16 r_{2}}{r_{1}}|z|, \quad|z| \leqslant \frac{r_{1}}{16} .
$$

We now assume that $a=1 / 2$. Let

$$
\sigma_{R}:=t(\log R)=\sup \left\{t<T: f_{t}^{\prime}(\infty)>1 / R\right\}
$$

For $t<T$, let $\widetilde{K}_{t}$ be the union of [0,1] with $K_{t}$ and with the reflection of $K_{t}$ about the real axis. Observe that $f_{t}$ extends conformally to a map $f_{t}: \mathbb{C} \backslash \widetilde{K}_{t} \rightarrow \mathbb{C} \backslash[0,1]$. Applying (4.1) to $F_{t}(z):=1 / f_{t}(1 / z)$ gives for all $t<T$,

$$
\frac{1}{4} \operatorname{rad}\left(\widetilde{K}_{t}\right) \leqslant F_{t}^{\prime}(0)=f_{t}^{\prime}(\infty)^{-1} \leqslant 4 \operatorname{rad}\left(\widetilde{K}_{t}\right),
$$


where rad denotes the radius with respect to the origin, i.e., $\operatorname{rad}(A):=\sup \{|z|: z \in A\}$. Since $R-(1 / 2) \leqslant \operatorname{rad}\left(\widetilde{K}_{\tau}\right) \leqslant R+(1 / 2)$, this gives

$$
\tau_{R / 4-1 / 2} \leqslant \sigma_{R} \leqslant \tau_{4 R+1 / 2}
$$

for all $R$ such that $\sigma_{R}<T$.

If $z \in A_{64 R}$, then (4.2) implies for $R>2$

$$
3 \leqslant \frac{64 R-(1 / 2)}{16(R+(1 / 2))} \leqslant\left|f_{\tau}(z)\right|=\left|F_{\tau}(1 / z)\right|^{-1} \leqslant \frac{16(64 R+(1 / 2))}{R-(1 / 2)} \leqslant 1999 .
$$

In particular,

$$
f_{\tau}\left(A_{64 R}\right) \subset V_{2000} \backslash V_{2} .
$$

For all $t<T$, let $L_{1}(t)$ be the length of the image of $I_{1}(t)$ under $f_{t}$, and let $L_{2}(t)$ be the length of the image of $I_{2}(t)$ under $f_{t}$. Recall that $Z_{t}=Y_{s(t)}$, and note that $\partial_{t} \log f_{t}^{\prime}(x)$ is monotone decreasing in $x$ when $f_{t}(x) \leqslant Z_{t}$, and monotone increasing for $f_{t}(x) \geqslant Z_{t}$, because

$$
\begin{aligned}
\partial_{t} \log f_{t}^{\prime}(x)+\partial_{t} \log \left(g_{t}(1)-g_{t}(0)\right) & =\partial_{t} \log g_{t}^{\prime}(x)=\frac{\partial_{x} \partial_{t} g_{t}(x)}{g_{t}^{\prime}(x)} \\
& =\frac{-2}{\left(g_{t}(x)-W_{t}\right)^{2}}=\frac{-2}{\left(f_{t}(x)-Z_{t}\right)^{2}\left(g_{t}(1)-g_{t}(0)\right)^{2}} .
\end{aligned}
$$

Therefore, $f_{t}^{\prime}(x) \leqslant f_{t}^{\prime}(0)$ for $x \in I_{1}(t)$ and $f_{t}^{\prime}(x) \leqslant f_{t}^{\prime}(1)$ for $x \in I_{2}(t)$. Consequently, $L_{1}(t) \leqslant f_{t}^{\prime}(0) / 2$ and $L_{2}(t) \leqslant f_{t}^{\prime}(1) / 2$.

Conformal invariance implies that

$$
\ell\left(A_{64 R}, I_{1}(\tau) ; \mathbb{H} \backslash \mathfrak{K}_{R}\right)=\ell\left(f_{\tau}\left(A_{64 R}\right), f_{\tau}\left(I_{1}(\tau)\right) ; \mathbb{H}\right) .
$$

If $I$ is any subinterval of $[-1,2]$, then it is straightforward to show that

$$
\exp \left(-\ell\left(A_{2}, I ; \mathbb{H}\right)\right) \asymp \text { length }(I) \asymp \exp \left(-\ell\left(A_{2000}, I ; \mathbb{H}\right)\right)
$$

Hence by comparison (provided $\tau<T$ ),

$$
\exp \left(-\ell\left(A_{64 R}, I_{1}(\tau) ; \mathbb{H} \backslash \mathfrak{K}_{R}\right)\right) \asymp L_{1}(\tau) \leqslant f_{\tau}^{\prime}(0) / 2,
$$

and similarly for $I_{2}$. Note that

$$
\mathfrak{L}_{1}(R) \leqslant \ell\left(A_{64 R}, I_{1}\left(\tau_{R}\right) ; \mathbb{H} \backslash \mathfrak{K}_{R}\right) \leqslant \ell\left(A_{64 R}, I_{1}\left(\tau_{64 R}\right) ; \mathbb{H} \backslash \mathfrak{K}_{64 R}\right)=\mathfrak{L}_{1}(64 R) .
$$

Hence,

$$
\mathbf{E}\left[1_{\left\{\tau_{64 R}<T\right\}} \exp \left(-w_{1} \mathfrak{L}_{1}(64 R)-w_{2} \mathfrak{L}_{2}(64 R)\right)\right] \leqslant c_{2} R^{-\lambda}
$$

follows from Theorem 3.1, (4.4), and (4.5). 
For the other direction, since $f_{t}^{\prime}$ is monotone decreasing on $(-\infty, 1 / 2) \backslash K_{t}$, if $\tau<T$, then

$$
\operatorname{length}\left(f_{\tau}([-1,1 / 2] \backslash \mathfrak{K})\right) \geqslant f_{\tau}^{\prime}(0)
$$

and

$$
\text { length }\left(f_{\tau}([1 / 2,2] \backslash \mathfrak{K})\right) \geqslant f_{\tau}^{\prime}(1) \text {. }
$$

Hence, when $\tau<T$,

$$
\begin{aligned}
f_{\tau}^{\prime}(0) & \leqslant \operatorname{length}\left(f_{\tau}\left([-1,1 / 2] \backslash \mathfrak{K}_{R}\right)\right) \\
& \asymp \exp \left(-\ell\left(A_{64 R},[-1,1 / 2] ; \mathbb{H} \backslash \mathfrak{K}_{R}\right)\right) \\
& \leqslant \exp \left(-\ell\left(A_{R},[-1,1 / 2] ; \mathbb{H} \backslash \mathfrak{K}_{R}\right)\right),
\end{aligned}
$$

and similarly for $f_{\tau}^{\prime}(1)$. Scale invariance of $S L E_{\kappa}$ tells us that the distribution of $\ell\left(A_{R},[-1,1 / 2] ; \mathbb{H} \backslash \mathfrak{K}_{R}\right)$ is the same as the distribution of $\ell\left(A_{R / 3},[0,1 / 2] ; \mathbb{H} \backslash \mathfrak{K}_{R / 3}\right)$. Combining this with Theorem 3.1 then readily shows that

$$
\mathbf{E}\left[1_{\left\{\tau<T_{R / 3}\right\}} \exp \left(-w_{1} \mathfrak{L}_{1}(R / 3)-w_{2} \mathfrak{L}_{2}(R / 3)\right)\right] \geqslant c_{3} R^{-\lambda},
$$

and completes the proof of Theorem 4.1.

\section{The universality argument}

Let $\mu_{R}$ denote the Brownian excursion measure in the domain $V_{R}$, and let $B$ denote an excursion. (See $[6,2,3]$ for the definition of the excursion measures on simply connected domain and the link with the Brownian intersection exponents.) Let $\mathcal{Q}_{B}$ denote the event that the initial point $B(0)$ of $B$ is in $(0,1 / 2)$, and the terminal point in $A_{R}$. On this event, let $\mathfrak{L}$ be the $\pi$-extremal distance from $[0, B(0)]$ to $A_{R}$ in $V_{R} \backslash B$, and let $\mathfrak{L}_{B}$ be the $\pi$-extremal distance from $[B(0), 1]$ to $A_{R}$ in $V_{R} \backslash B$. Then for all $w, w^{\prime}>0$, when $R \rightarrow \infty$,

$$
\int_{\mathcal{Q}_{B}} \exp \left(-w \mathfrak{L}-w^{\prime} \mathfrak{L}_{B}\right) \mathrm{d} \mu_{R}(B) \approx R^{-\widetilde{\xi}\left(w, 1, w^{\prime}\right)} .
$$

Let $\phi=\phi_{B}$ be the conformal map from the component $X=X_{B}$ of $V_{R} \backslash B$ whose boundary contains $[B(0), 1]$ to a semi-disk $V_{\widetilde{R}(B)}$ such that $\phi$ takes $\partial X \cap[B(0), 1]$ onto $[0,1]$ and takes $\partial X \cap A_{R}$ onto $A_{\widetilde{R}(B)}$. Set $\widetilde{\mathfrak{L}}_{B}:=\log \widetilde{R}(B)$. Note that when $R \rightarrow \infty$,

$$
\widetilde{\mathfrak{L}}_{B}=\mathfrak{L}_{B}+\mathrm{O}(1) .
$$

Hence, for all $w, w^{\prime}>0$, when $R \rightarrow \infty$,

$$
\int_{\mathcal{Q}_{B}} \exp \left(-w \mathfrak{L}-w^{\prime} \widetilde{\mathfrak{L}}_{B}\right) \mathrm{d} \mu_{R}(B) \approx R^{-\widetilde{\xi}\left(w, 1, w^{\prime}\right)} .
$$


We will need a lemma saying that we can restrict ourselves to the case where $B(0)<1 / 2$ and the conformal map $\phi$ does not push $1 / 2$ too close to 0 . More precisely, let $\mathcal{H}$ denote the event that $\mathcal{Q}_{B}$ holds and $\phi(1 / 2) \in[1 / 20,19 / 20]$.

LEMmA 5.1. - For all $w, w^{\prime} \geqslant 0$, as $R \rightarrow \infty$,

$$
\int_{\mathcal{H}} \exp \left(-w \mathfrak{L}-w^{\prime} \mathfrak{L}_{B}\right) \mathrm{d} \mu_{R}(B) \approx R^{-\widetilde{\xi}\left(w, 1, w^{\prime}\right)} .
$$

Proof. - Let $M:=\{z \in \mathbb{H}:|z-1| \leqslant 9 / 10\}$, and let $\mathcal{M}$ be the event $B \cap M=\emptyset$. We first show that $\mathcal{Q}_{B} \cap \mathcal{M} \subset \mathcal{H}$. Indeed, extend $\phi$ to $\left\{\bar{z}: z \in X_{B}\right\}$, by Schwarz reflection. Since $\phi(1)=1$ and $\phi\left(X_{B}\right) \supset X_{B}$, it follows from the Schwarz Lemma that $\phi(x) \leqslant x$ for all $x \in[B(0), 1]$. In particular $\phi(1 / 2) \leqslant 1 / 2$. Let $\psi$ be the conformal map from the disk $\{z:|z-1|<9 / 10\}$ onto $\mathbb{C} \backslash(-\infty, 0]$ such that $\psi(1)=1$ and $\psi^{\prime}(1)>0$. The Schwarz Lemma also shows that $\phi(x) \geqslant \psi(x)$ for all $x \in[1 / 10,1]$. Since $\psi(z)=(10 z-1)^{2} /(10 z-19)^{2}$, it follows that $\phi(1 / 2) \geqslant \psi(1 / 2)>1 / 20$. This proves $\mathcal{Q}_{B} \cap \mathcal{M} \subset \mathcal{H}$.

On the event $\mathcal{Q}_{B} \cap \mathcal{M}$, let $\mathfrak{L}_{B}^{\prime}:=\ell\left([B(0), 1 / 10], A_{R} ; V_{R} \backslash(B \cup M)\right)$ be the $\pi$-extremal distance from $[B(0), 1 / 10]$ to $A_{R}$ in $V_{R} \backslash(B \cup M)$. Let $L^{\prime}$ be the $\pi$-extremal distance from [0,1/10] to $A_{R}$ in $V_{R} \backslash M$. It is clear that $\log R \leqslant L^{\prime} \leqslant \log R+\mathrm{O}(1)$. Consequently, by the restriction property and conformal invariance for Brownian excursions, it follows that

$$
\int_{\mathcal{M} \cap \mathcal{Q}_{B}} \exp \left(-w \mathfrak{L}-w^{\prime} \mathfrak{L}_{B}^{\prime}\right) \mathrm{d} \mu_{R}(B) \approx R^{-\widetilde{\xi}\left(w, 1, w^{\prime}\right)} .
$$

Observe that $\mathfrak{L}_{B} \leqslant \mathfrak{L}_{B}^{\prime}$ and that

$$
\int_{\mathcal{Q}_{B}} \exp \left(-w \mathfrak{L}-w^{\prime} \mathfrak{L}_{B}\right) \mathrm{d} \mu_{R}(B) \approx R^{-\widetilde{\xi}\left(w, 1, w^{\prime}\right)} .
$$

Since the left hand side of (5.1) is between the left hand sides of (5.2) and (5.3), the lemma follows.

Proof of Theorem (1.1). - Let $\mu_{R}$ denote the Brownian excursion measure in the domain $V_{R}$, and let $B$ be an excursion. Let $\mathbf{P}_{R}$ denote the law of $S L E_{6}$ in $V_{R}$ started from $1 / 2$, and let $\mathfrak{K}$ be as in the previous section. Let $\mathcal{Q}_{B}$ be the event that the initial point $B(0)$ of $B$ is in $[0,1 / 2]$ and the terminal point is in $A_{R}$, let $\mathcal{Q}_{\mathfrak{K}}$ be the event that $\mathfrak{K} \subset \mathbb{H} \cup[0,1]$, and let $\mathcal{Q}$ be the event $\mathcal{Q}_{\mathfrak{K}} \cap \mathcal{Q}_{B} \cap\{\mathfrak{K} \cap B=\emptyset\}$. On $\mathcal{Q}$ let

$$
\begin{aligned}
\mathfrak{L} & :=\ell\left([0, B(0)], A_{R} ; V_{R} \backslash B\right), \\
\mathfrak{L}_{B} & :=\ell\left([B(0), 1], A_{R} ; V_{R} \backslash B\right), \\
\mathfrak{L}_{\mathfrak{K}} & :=\ell\left([0,1 / 2], A_{R} ; V_{R} \backslash \mathfrak{K}\right), \\
\mathfrak{L}^{\prime} & :=\ell\left([B(0), 1 / 2], A_{R} ; V_{R} \backslash(\mathfrak{K} \cup B)\right), \\
\mathfrak{L}^{\prime \prime} & :=\ell\left([1 / 2,1], A_{R} ; V_{R} \backslash \mathfrak{K}\right) .
\end{aligned}
$$


We determine the asymptotics as $R \rightarrow \infty$ of $\mathbf{E}\left[1_{\mathcal{Q}} \exp \left(-w \mathfrak{L}-w^{\prime} \mathfrak{L}^{\prime}-w^{\prime \prime} \mathfrak{L}^{\prime \prime}\right)\right]$ in two different ways.

Given $B \in \mathcal{Q}_{B}$, we may map $X_{B}$ onto $V_{\exp \left(\widetilde{\mathfrak{L}}_{B}\right)}$ by $\phi$. By conformal invariance of $S L E_{6}$, the restriction property of $S L E_{6}$ (see [2]) and Theorem 4.1, it follows that

$$
\mathbf{E}\left[\exp \left(-w^{\prime} \mathfrak{L}^{\prime}-w^{\prime \prime} \mathfrak{L}^{\prime \prime}\right) \mid B\right] \leqslant c \exp \left(-\lambda_{6}\left(w^{\prime}, w^{\prime \prime}\right) \widetilde{\mathfrak{L}}_{B}\right) .
$$

Hence,

$$
\begin{aligned}
& \int_{\mathcal{Q}_{B}} \int_{\mathcal{Q}_{\mathfrak{K}}} 1_{\mathcal{Q}} \exp \left(-w \mathfrak{L}-w^{\prime} \mathfrak{L}^{\prime}-w^{\prime \prime} \mathfrak{L}^{\prime \prime}\right) \mathrm{d} \mathbf{P}_{R}(\mathfrak{K}) \mathrm{d} \mu_{R}(B) \\
& \quad \leqslant c \int_{\mathcal{Q}_{B}} \exp \left(-w \mathfrak{L}-\lambda_{6}\left(w^{\prime}, w^{\prime \prime}\right) \mathfrak{L}_{B}\right) \mathrm{d} \mu_{R}(B) \\
& \quad \approx R^{-\widetilde{\xi}\left(w, 1, \lambda_{6}\left(w^{\prime}, w^{\prime \prime}\right)\right)} .
\end{aligned}
$$

For the other direction, by Theorem 4.1 and by Lemma 5.1, we have

$$
\begin{aligned}
& \int_{\mathcal{Q}_{B}} \int_{\mathcal{Q}_{\mathfrak{K}}} 1_{\mathcal{Q}} \exp \left(-w \mathfrak{L}-w^{\prime} \mathfrak{L}^{\prime}-w^{\prime \prime} \mathfrak{L}^{\prime \prime}\right) \mathrm{d} \mathbf{P}_{R}(\mathfrak{K}) \mathrm{d} \mu_{R}(B) \\
& \geqslant \int_{\mathcal{H}} \int_{\mathcal{Q}_{\mathfrak{K}}} 1_{\mathcal{Q}} \exp \left(-w \mathfrak{L}-w^{\prime} \mathfrak{L}^{\prime}-w^{\prime \prime} \mathfrak{L}^{\prime \prime}\right) \mathrm{d} \mathbf{P}_{R}(\mathfrak{K}) \mathrm{d} \mu_{R}(B) \\
& \geqslant c^{\prime} \int_{\mathcal{H}} \exp (-w \mathfrak{L}) \exp \left(-\lambda_{6}\left(w^{\prime}, w^{\prime \prime}\right) \widetilde{\mathfrak{L}}_{B}\right) \mathrm{d} \mu_{R}(B) \\
& \approx R^{-\widetilde{\xi}\left(w, 1, \lambda_{6}\left(w^{\prime} w^{\prime \prime}\right)\right)} .
\end{aligned}
$$

We may therefore conclude that

$$
\int_{\mathcal{Q}_{B}} \int_{\mathcal{Q}_{\mathfrak{K}}} 1_{\mathcal{Q}} \exp \left(-w \mathfrak{L}-w^{\prime} \mathfrak{L}^{\prime}-w^{\prime \prime} \mathcal{L}^{\prime \prime}\right) \mathrm{d} \mathbf{P}_{R}(\mathfrak{K}) \mathrm{d} \mu_{R}(B) \approx R^{-\widetilde{\xi}\left(w, 1, \lambda_{6}\left(w^{\prime}, w^{\prime \prime}\right)\right)} .
$$

On the other hand, by conformal invariance and the restriction property of the Brownian excursions, given $\mathfrak{K} \in \mathcal{Q}_{\mathfrak{K}}$, we have

$$
\int 1_{\mathcal{Q}} \exp \left(-w \mathfrak{L}-w^{\prime} \mathfrak{L}^{\prime}\right) \mathrm{d} \mu_{R}(B) \approx \exp \left(-\widetilde{\xi}\left(w, 1, w^{\prime}\right) \mathfrak{L}_{\mathfrak{K}}\right) .
$$

Consequently, Theorem 4.1 gives

$$
\begin{aligned}
& \int_{\mathcal{Q}_{\mathfrak{K}}} \int_{\mathcal{Q}_{B}} 1_{\mathcal{Q}} \exp \left(-w \mathfrak{L}-w^{\prime} \mathfrak{L}^{\prime}-w^{\prime \prime} \mathfrak{L}^{\prime \prime}\right) \mathrm{d} \mu_{R}(B) \mathrm{d} \mathbf{P}_{R}(\mathfrak{K}) \\
& \quad \approx \int_{\mathcal{Q}_{\mathfrak{K}}} \exp \left(-\widetilde{\xi}\left(w, 1, w^{\prime}\right) \mathfrak{L}_{\mathfrak{K}}-w^{\prime \prime} \mathfrak{L}^{\prime \prime}\right) \mathrm{d} \mathbf{P}_{R}(\mathfrak{K}) \\
& \quad \approx R^{-\lambda_{6}\left(\widetilde{\xi}\left(w, 1, w^{\prime}\right), w^{\prime \prime}\right)} .
\end{aligned}
$$


Comparing with (5.4) gives

$$
\lambda_{6}\left(\widetilde{\xi}\left(w, 1, w^{\prime}\right), w^{\prime \prime}\right)=\widetilde{\xi}\left(w, 1, \lambda_{6}\left(w^{\prime}, w^{\prime \prime}\right)\right) .
$$

Define $y\left(w^{\prime}\right):=\lim _{w \searrow 0} \lambda_{6}\left(w, w^{\prime}\right)$. First let $w \searrow 0$ and $w^{\prime} \searrow 0$ in (5.5). Recall that $\left(w, w^{\prime}\right) \mapsto \widetilde{\xi}\left(w, 1, w^{\prime}\right)$ is continuous at $(0,0)$ (see e.g., [5]), so that for all $w^{\prime \prime} \geqslant 0$

$$
\lambda_{6}\left(1, w^{\prime \prime}\right)=\widetilde{\xi}\left(1, y\left(w^{\prime \prime}\right)\right),
$$

which shows that $\widetilde{\xi}(1, v)=\lambda_{6}\left(1, y^{-1}(v)\right)$ in the case where $v \geqslant 1$ (we also derived this result in [2]).

Now $w \searrow 0$ and $w^{\prime \prime} \searrow 0$ in (5.5) gives

$$
y\left(\widetilde{\xi}\left(1, w^{\prime}\right)\right)=\widetilde{\xi}\left(1, y\left(w^{\prime}\right)\right) .
$$

Combining this with (5.6) and the explicit expression for $\lambda_{6}$ shows that for all $v>0$

$$
\widetilde{\xi}(1, v)=y^{-1}\left(\lambda_{6}(1, v)\right)=y(v) .
$$

Finally, letting $w^{\prime} \searrow 0$ in (5.5) shows that $\tilde{\xi}\left(w, 1, y\left(w^{\prime \prime}\right)\right)=\lambda_{6}\left(\widetilde{\xi}(w, 1), w^{\prime \prime}\right)$, which gives

$$
\widetilde{\xi}\left(w, 1, \widetilde{\xi}\left(1, w^{\prime \prime}\right)\right)=\lambda_{6}\left(y(w), w^{\prime \prime}\right) .
$$

The cascade relations (1.1) and (5.7) applied to the left hand side imply

$$
\widetilde{\xi}\left(w, w^{\prime \prime}\right)=y^{-1} \circ y^{-1} \circ \lambda_{6}\left(y(w), w^{\prime \prime}\right) .
$$

Via further applications of the cascade relations, this leads to the explicit expression for all $\widetilde{\xi}\left(w_{1}, \ldots, w_{k}\right)$.

Proof of Theorem 1.2. - By (1.2) and Theorem 1.1, it suffices to derive the value of $\xi(1, w, 1, w)$ for all $w>0$. This is a simple combination of Theorem 4.1, the relation between radial and chordal $S L E_{6}$ (see [3]), and the computation of exponents for radial $S L E_{6}$ (see [3]). The proof is essentially the same as in the final section of [3]. One has to consider (for small $r>0$ ) a Brownian excursion $B$ in the annulus $A_{r}=\{z: r<|z|<1\}$ and an independent radial $S L E_{6}$ started at 1 (growing towards 0 ) stopped when it hits the circle of radius $r$, and the event $\mathcal{C}$ that they both cross the annulus without intersecting each other. Define $\mathfrak{L}$ and $\mathfrak{L}^{\prime}$ to be the two $\pi$-extremal distances between the two circles in each of the two connected components of $A_{r} \backslash(B \cup \mathfrak{K})$ that cross the annulus. The result is derived by estimating the integral of $\exp \left(-w \mathfrak{L}-w \mathfrak{L}^{\prime}\right)$ in two ways. First, fixing $B$ and applying Theorem 4.1 and [3, Lemma 5.5] gives the exponent $\xi\left(1, \lambda_{6}(w, w)\right)$; this is equal to $\xi(1, \widetilde{\xi}(w, 1, w))=\xi(1,1, w, w)$ by Theorem 1.1 . On the other hand, if we first fix $\mathfrak{K}$ and use the radial $S L E_{6}$ exponents derived in [3] and the value of $\widetilde{\xi}(w, 1, w)$ (from Theorem 1.1), we can compute explicitly the exponent. Since this is almost word for word the same argument as in the final section of [3], we safely leave the details to the reader. 
The fact that $(1.4)$ is valid for all $x \geqslant \widetilde{\xi}(1,1)$ is also an immediate corollary of (1.4) and the analyticity result from [4]. Consequently, Theorem 1.2 also follows from [4] and Theorem 1.1.

\section{REFERENCES}

[1] Ahlfors L.V., Conformal Invariants, Topics in Geometric Function Theory, McGraw-Hill, New York, 1973.

[2] Lawler G.F., Schramm O., Werner W., Values of Brownian intersection exponents I: Halfplane exponents, Acta Mathematica (1999), to appear.

[3] Lawler G.F., Schramm O., Werner W., Values of Brownian intersection exponents II: Plane exponents, Acta Mathematica (2000), to appear.

[4] Lawler G.F., Schramm O., Werner W., Analyticity of planar Brownian intersection exponents, Acta Mathematica (2000), to appear.

[5] Lawler G.F., Werner W., Intersection exponents for planar Brownian motion, Ann. Probab. 27 (1999) 1601-1642.

[6] Lawler G.F., Werner W., Universality for conformally invariant intersection exponents, J. European Math. Soc. 2 (2000) 291-328.

[7] Schramm O., Scaling limits of loop-erased random walks and uniform spanning trees, Israel J. Math. 118 (2000) 221-228. 